\title{
BLOCH'S THEOREM FOR REAL VARIABLES
}

S. BOCHNER

The famous theorem of Bloch states that every function

$$
F(z)=z+a_{2} z^{2}+\cdots
$$

of the complex variable $z$ in the unit-circle $|z| \leqq 1$ maps some subdomain of the unit-circle univalently onto a circle of a fixed radius whose size is independent of $f(z)$. The purpose of the present paper is to point out the following generalization to $n$ real variables, $n \geqq 2$.

Theorem 1. Corresponding to any integer $n, n=1,2,3, \cdots$, and any positive constant $K, K>0$, there exists a positive radius

$$
R_{0}=R_{0}(n, K), \quad R_{0}>0,
$$

having the following property:

If the real functions

$$
u_{i}=f_{i}\left(x_{1}, \cdots, x_{n}\right), \quad i=1,2, \cdots, n,
$$

are defined and solutions of the Laplace equation

$$
\frac{\partial^{2} f}{\partial x_{1}^{2}}+\cdots+\frac{\partial^{2} f}{\partial x_{n}^{2}}=0
$$

in a neighborhood of the sphere

$$
x_{1}^{2}+\cdots+x_{n}^{2} \leqq 1
$$

if their Jacobian

$$
J\left(x_{1}, \cdots, x_{n}\right)=\frac{\partial\left(f_{1}, \cdots, f_{n}\right)}{\partial\left(x_{1}, \cdots, x_{n}\right)}
$$

satisfies the decisive relation

$$
\sum_{i, j=1}^{n}\left(\frac{\partial f_{i}}{\partial x_{j}}\right)^{2} \leqq K \cdot\left|J\left(x_{1}, \cdots, x_{n}\right)\right|^{2 / n}
$$

in (4), and if

$$
J(0, \cdots, 0)=1,
$$

then there exists in (4) an open set $S$ such that the functions (2) are a one-

Received by the editors May 6, 1946, 
to-one transformation of $S$ onto a sphere of radius $R_{0}$ in the Euclidean space of the variables $\left(u_{1}, \cdots, u_{n}\right)$.

The decisive restriction (6) can be easily interpreted. If we introduce the differential form

$$
d s^{2}=g_{i j}(x) d x_{i} d x_{i}
$$

where

$$
g_{i j}(x)=\sum_{m} \frac{\partial f_{m}}{\partial x_{i}} \cdot \frac{\partial f_{m}}{\partial x_{j}},
$$

then in the ellipsoid $g_{i j}(x) \xi_{i} \xi_{j}=1$ the quotient of the longest over the shortest axis shall have a finite bound which is independent of $x$; if, however, the determinant of $g_{i j}$ vanishes at a point, then all numbers $\partial f_{m} / \partial x_{i}$ shall vanish there separately. This is a generalization of conformal transformations, for which all axes have the same length at any point.

For $n=2$ the significance of this generalization has been brought to light in the work of Ahlfors ${ }^{1}$ when he proved that Picard's theorem on exceptional values will remain in force for this type of transformation. For $n \geqq 3$, our condition may turn out to be of even greater significance. On the one hand there are no strictly conformal transformations other than trivial ones (inversions in spheres). But on the other hand, general analytic transformations in several complex variables of the form

$$
w_{l}=F_{l}\left(z_{1}, \cdots, z_{k}\right), \quad l=1, \cdots, k ; n=2 k,
$$

do not obey our restriction for $k \geqq 2$, which fact may be said to be responsible for the complete breakdown of Picard's theorem in that case.

As for our additional requirement that each component $f_{i}(x)$ shall be a solution of (3), it will be evident from the course of the proof that it could be considerably toned down. However, we are drawing up the condition in this rigid form in order to point up an analogy to the case of complex variables. For one complex variable, (3) requires as in the classical case that each component of (1) separately shall be a solution of the Laplace equation. However, in lieu of the rigorous rela-

1 Compare the account in R. Nevanlinna, Eindeutige analytische Funktiones, p. 343. There were also earlier efforts in that direction. See, for instance, H. Groetzsch, Ueber möglichst konforme Abbildungen von schlichten Bereichen, Berichte uber der Verhandlungen der K. Sächsischen Akademie der Wissenschaften zu Leipzig, Mathematische-Physische Klasse, vol. 84 (1932) pp. 114-120. 
tion between the two components as implied in the Cauchy-Riemann equation we now have the much milder condition (6). For several complex variables, analyticity implies in particular the relations

$$
\frac{\partial^{2} f_{i}}{\partial x_{l}^{2}}+\frac{\partial^{2} f_{i}}{\partial y_{l}^{2}}=0, \quad l=1, \cdots, k,
$$

for each pair $\left(x_{l}, y_{l}\right)$ separately, whereas our present condition (3) replaces this by the summary requirement

$$
\sum_{l=1}^{k}\left(\frac{\partial^{2} f_{i}}{\partial x_{l}^{2}}+\frac{\partial^{2} f_{i}}{\partial y_{l}^{2}}\right)=0
$$

thus creating a situation which is even more general than the case of one complex variable would indicate.

For the proof of Theorem 1 we introduce the functions

$$
M(r)=\max _{x_{1}^{2}+\cdots+x_{n}^{2} \leqq r^{2}}\left|J\left(x_{1}, \cdots, x_{n}\right)\right|^{1 / n}
$$

and

$$
\phi(r)=(1-r) M(r) .
$$

Since $\phi(0)=1$ and $\phi(1)=0$, there exists a number $r^{0}$ such that

$$
\phi\left(r^{0}\right)=1, \quad \phi(r)<1 \text { for } r^{0}<r \leqq 1 .
$$

Also, there exists a point

$$
x^{0}=\left(x_{1}^{0}, \cdots, x_{n}^{0}\right)
$$

for which $\left(x_{1}\right)^{2}+\cdots+\left(x_{n}\right)^{2}$ is precisely equal to $\left(r^{0}\right)^{2}$ and such that

$$
J\left(x_{1}^{0}, \cdots, x_{n}^{0}\right)^{1 / n}=M\left(r^{0}\right)=\frac{1}{1-r^{0}} .
$$

Now introduce the functions

$$
g_{i}\left(\xi_{1}, \cdots, \xi_{n}\right)=\frac{f_{i}\left(x_{m}^{0}+\rho \xi_{m}\right)-f_{i}\left(x_{m}^{0}\right)}{\rho\left|J\left(x_{m}^{0}\right)\right|^{1 / n}}, \quad i=1, \cdots, n,
$$

where

$$
\rho=\frac{1-r^{0}}{2}
$$

and since for

$$
\xi_{1}^{2}+\cdots+\xi_{n}^{2} \leqq 1
$$


we have

$$
\sum_{m=1}^{n}\left(x_{m}^{0}+\rho \xi_{m}\right)^{2} \leqq\left(\frac{1+r^{0}}{2}\right)^{2},
$$

they will exist in the unit sphere (11). We also introduce their Jacobian

$$
G\left(\xi_{1}, \cdots, \xi_{n}\right)=\frac{\partial\left(g_{1}, \cdots, g_{n}\right)}{\partial\left(\xi_{1}, \cdots, \xi_{n}\right)},
$$

and we list the following properties:

(i) All functions $g_{i}(\xi)$ are again solutions of the Laplacean $\partial^{2} g / \partial \xi_{1}^{2}$ $+\cdots+\partial^{2} g / \partial \xi_{n}^{2}=0$.

(ii) $G(0, \cdots, 0)=1$.

(iii) $G\left(\xi_{1}, \cdots, \xi_{n}\right) \leqq 2^{n}$ for $\xi$ in (11).

(iv) $\sum_{i, j=1}^{n}\left(\partial g_{i} / \partial \xi_{j}\right)^{2} \leqq K\left|G\left(\xi_{1}, \cdots, \xi_{n}\right)\right|^{2 / n} \leqq 4 K$.

We note that property (iii) follows from

$$
\begin{aligned}
\left|\frac{\partial\left(g_{1}, \cdots, g_{n}\right)}{\partial\left(\xi_{1}, \cdots, \xi_{n}\right)}\right| & =\frac{\left|J\left(x_{1}, \cdots, x_{n}\right)\right|}{\left|J\left(x_{1}^{0}, \cdots, x_{n}^{0}\right)\right|} \leqq\left(\frac{M\left(\left(1+r^{0}\right) / 2\right)}{M\left(r^{0}\right)}\right)^{n} \\
& \leqq\left(\frac{2}{1-r^{0}}\left(1-r^{0}\right)\right)^{n}=2^{n}
\end{aligned}
$$

We are now going to make use of property (i). Since the first partial derivatives $\partial g_{i} / \partial \xi_{j}$ are themselves solutions of the Laplacean, it follows from Poisson's integral for harmonic functions that corresponding to $n$ and $K$ there exists a constant $L=L(n, K)$ such that the validity of property (iv) in (11) will imply the further property

$$
\sum_{i, j, k=1}^{n}\left(\frac{\partial^{2} g_{i}}{\partial \xi_{j} \partial \xi_{k}}\right)^{2} \leqq L
$$

in

$$
\xi_{1}^{2}+\cdots+\xi_{n}^{2} \leqq 1 / 2 .
$$

However, properties (ii), (iv) and (v), together with

$$
g_{\imath}(0, \cdots, 0)=0, \quad i=1, \cdots, n,
$$

lead to the following conclusion. There exists a radius $R_{1}=R_{1}(n, K, L)$ $\equiv R_{1}(n, K)$ such that on some subset $B$ of (12)-the subset depending on the given functions $g_{i}(\xi)$-the functions

$$
\eta_{i}=g_{i}\left(\xi_{1}, \cdots, \xi_{n}\right)
$$


are a one-to-one transformation of $B$ into the sphere

$$
\eta_{1}^{2}+\cdots+\eta_{n}^{2} \leqq R_{1}^{2} \text {. }
$$

This is a "uniform" version of the theorem on implicit functions and can be derived from the Kronecker integral or in some other fashion. ${ }^{2}$

Returning now to the original functions (2), we see from (10) that these functions will transform a subdomain $B$ of (4) into a sphere of radius

$$
R_{0}=R_{1} \cdot \rho\left|J\left(x_{m}^{0}\right)\right|^{1 / n} .
$$

However, $\rho\left|J\left(x_{m}{ }^{0}\right)\right|^{1 / n}=\left(\left(1-r_{0}\right) / 2\right) \cdot\left(1 /\left(1-r_{0}\right)\right)=1 / 2$, and this completes the proof of Theorem 1 .

THEOREM 2. If all assumptions of Theorem 1 are fulfilled in the sphere $x_{1}^{2}+\cdots+x_{n}^{2} \leqq \lambda^{2}$, then the map contains a sphere of radius $R$ $=\lambda \cdot R_{0}(n, K)$.

This follows from Theorem 1 , if we replace $f_{i}(x)$ by $\lambda f_{i}(x / \lambda)$.

Hence we conclude:

THEOREM 3. If all assumptions of Theorem 1 are fulfilled in the entire space, then the map contains spheres of arbitrarily large radius.

Princeton University

See, for instance, the first chapter in Caratheodory's Variationsrechnung. 